\title{
Lower blood malondialdehyde is associated with past pesticide exposure: findings in Gulf War illness and healthy controls
}

\author{
Beatrice Alexandra Golomb ${ }^{1 *} \mathbb{0}$, Sridevi Devaraj ${ }^{2}$, Alexis K. Messner ${ }^{1,3}$, Hayley Jean Koslik,4, Jun Hee Han ${ }^{1}$ and \\ Barnabas Yik ${ }^{1,5}$
}

\begin{abstract}
Background: Malondialdehyde (MDA) is a candidate general marker of oxidative stress (OS). We sought to assess the relation of MDA to Gulf War illness (GWI) and to a variety of exposures.

Methods: This is an observational study involving subjects from Southern California recruited from October 2011 to May 2014. MDA was assessed in 81 participants (41 GWI-cases, 40 controls). General and Gulf-specific exposures were elicited. MDA case-control comparison was restricted to 40 matched pairs. The potential association between MDA and exposures was assessed using regression analyses. Gulf-specific exposures were incorporated into a case-specific model.

Results: Plasma MDA was significantly lower in GWI-cases than controls. Composite pesticide and fuel-solvent exposures negatively predicted MDA in the total sample, as well as in the analyses that included either GWI-cases or controls only. Self-reported exposure to organophosphate (OP) nerve gas was a strong predictor for lower MDA level in veterans with GWI.
\end{abstract}

Conclusion: Past pesticide exposures predicted lower MDA in both veterans with GWI and in healthy controls.

Keywords: Malondialdehyde, Oxidative stress, Free radical, Gulf War veterans, Gulf War illness, Pesticide

\section{Background}

Malondialdehyde (MDA) is a measure of peroxidized lipids, and has been considered as a good candidate general marker of oxidative stress (OS) by an NIH working group based on assessments involving carbon tetrachloride $\left(\mathrm{CCl}_{4}\right)$ : "It is concluded that measurements of MDA ... in plasma and urine ... are potential candidates for general biomarkers of oxidative stress" [1]. There is a need for information on how robust and generalizable MDA could serve as the marker of OS, both current and

*Correspondence: bgolomb@ucsd.edu

1 Department of Medicine, School of Medicine, University of California, San Diego, La Jolla, CA 92093, USA

Full list of author information is available at the end of the article past, in different settings and within groups with other signatures indicative of OS injury.

Ground operation in the 1990-1991 Persian Gulf War lasted only 4 days, and many personnel was not involved in actual combat, but approximately $1 / 3$ of the 700,000 US personnel deployed in the war later developed a variety of chronic health problems, now collectively termed "Gulf War illness (GWI)" [2, 3]. Causes of GWI are complex, but involves a range of new and unique environmental exposures, including oil fires, depleted uranium bombs, anthrax vaccine, the highest maximal number of multiple vaccines, and most notably acetylcholinesterase inhibiting agents, such as organophosphate (OP) and carbamate subclasses [4] and pesticides [5-8]. 
Many of the above-mentioned exposures cause OS [9]. OS is also implicated in some component symptoms of GWI [9]. In addition, mitochondrial impairment has been documented in veterans with GWI $[10,11]$. The relationship between MDA and impaired mitochondria is controversial. Some evidence suggests that mitochondrial impairment leads to more free radicals in the process of energy production [12], and thus increased MDA. Other studies, however, supported reduced MDA with mitochondrial impairment. (There is precedent for association between some exposures to increased OS in many studies [13,14], but decreased MDA under other circumstances [15].) Since membrane arachidonic acid relates positively to mitochondrial function [16, 17], and since impaired mitochondrial function has been reported in veterans with GWI $[10,11]$, membrane arachidonic acid might be depressed in veterans with GWI. (Arachidonic acid has been reported to inhibit mitochondrial function-so the relationship between lowered arachidonic acid and impaired mitochondrial function might reflect decreased arachidonic acid [18].) Moreover, reduced fatty acid beta-oxidation caused by bioenergetic impairment in GWI can lead to reduced oxidation of arachidonic acid and its products [19].

In the current study, we compared blood MDA concentration in veterans with GWI versus healthy controls, and examined the potential association between MDA with a variety of exposures.

\section{Methods}

This observational study in community-dwelling individuals in Southern California sought to compare blood malondialdehyde in cases versus controls, and to assess exposure relations to MDA. The study was approved by the UCSD Human Research Protections Program (HRPP). All subjects gave written informed consent to participate.

\section{Design, setting and participants}

This study was observational. A total of 81 subjects, including 41 veterans with GWI and 40 healthy controls, were recruited from October 2011 to May 2014. Among these subjects, 40 veterans with GWI and 40 healthy controls were matched in sex, age (within 4 years) and ethnicity (half match). Participants were communitydwelling individuals primarily from Southern California.

Cases To qualify as having GWI, subjects must have been deployed to the Gulf theater at any point between August 1990 and July 1991, and meet the Centers for Disease Control and Prevention (CDC) and Kansas inclusion criteria for GWI [20,21]. CDC criteria require symptoms for at least 6 months arising since the Gulf War in at least two of three domains of fatigue/sleep, mood-cognitive, and musculoskeletal [20]. The Kansas criteria require symptoms for at least 6 months arising during or after Gulf deployment in at least 3 of 6 domains of fatigue/ sleep, pain, neurological/cognitive/mood, respiratory, gastrointestinal, and dermatologic [21]. Symptoms in an individual domain must be at least moderate in severity and/or must be multiple [21]. The unmatched, 41st case was excluded from case-control comparisons, but included in analyses that predict MDA by exposures.

Controls Controls must be nonveterans, and meet neither Kansas nor CDC symptom inclusion criteria for GWI. Controls were also required not to meet Kansas exclusion criteria (i.e., not having other health conditions that could be confused for GWI, such as lupus or multiple sclerosis).

\section{Test-sets and sample size}

MDA was compared in two sets of 20 pairs each ("testsets"), with the unmatched 41st case in the second testset. The first test-set was conducted during the period of participant visits, and the second test-set following the conclusion of participant visits and sample collection. For paired testing, this sample provides $94 \%$ power to detect a 0.40 standard deviation effect size, with a two-sided alpha of 0.05 (G*Power, version 3.1.9.7, HHU, Düsseldorf, Germany-see Additional file 1 for citation information). For unpaired testing, the sample provides $80 \%$ power to detect 0.63 standard deviation effect size.

\section{Exposure assessment}

Participants completed surveys regarding environmental exposures in and out of the Gulf. Because of difficulties quantifying many types of exposure, self-rating was qualitative, rated as no (0), unsure (0.5), or yes (1).

\section{MDA assessment}

Plasma MDA concentration was determined using the MDA-586 method from Oxis International, based on the reaction of the chromogenic N-methyl-2-phenylindole (NMPI) with MDA at $45^{\circ} \mathrm{C}$. One molecule of MDA reacts with 2 molecules of NMP to yield a stable carbocyanine dye. Absorbance was detected at $586 \mathrm{~nm}$. Samples were archived at $-80{ }^{\circ} \mathrm{C}$ until analysis. Analysis of samples was conducted in two test-sets, as described above. Samples were shipped to the analysis site on dry ice with overnight shipping.

\section{$\mathrm{MDA}_{1}$}

To account for possible effects of archive time, a regression predicting MDA by time-to-test (i.e. archive time) and $\ln$ (time-to-test) was performed using controls only. For both cases and controls, $\mathrm{MDA}_{1}$ was calculated as the departure of measured MDA from predicted MDA by 
this regression. We calculated the departure of measured MDA from that predicted by time-to-test variablesthe portion of the variation in MDA not attributable to archive time.

\section{Composite variable creation}

Because of high collinearity among pesticide-herbicide and fuel-solvent exposures, with pesticide exposures showing the larger coefficients in MDA prediction, a composite pesticide variable was created. A score of 1 was assigned if exposure to any of OP, organochlorine, or lice treatment is present; a score of zero was assigned otherwise.

\section{Statistical analysis}

Continuous variables were compared using Student's $t$-test. Categorical variables were compared using the chi-squared test. There were no missing data on the outcome variable of MDA. MDA was compared in cases versus controls by Student's $t$-test $\left(\mathrm{MDA}_{1}\right)$ and regression with robust standard errors, to assess prediction of $\mathrm{MDA}_{1}$ by case status. Prediction of MDA (by exposures) employed regression analysis with robust (heteroskedasticity-independent) standard errors, excluding those who rated an exposure as "unsure." The analysis was conducted in the total sample, in cases, and in controls separately, with adjustment for the time variables. To address potential outcome bias that could arise from disparities in archive time, findings were adjusted for archive time and $\ln$ (archive time). In these regressions adjusted for time variables, MDA rather than $\mathrm{MDA}_{1}$ was used. The relative consistency of key findings was assessed separately for first versus second test-sets, and for cases and controls. The final exposure regressions in the total sample were adjusted for case status and test-set; and replicated in each test-set adjusted for case status. For analyses involving exposures that were rated as unsure, or (rarely) were unrated, the participant was dropped from the analysis except as stated. Missing data were not imputed, but no participants were missing MDA values, the composite pesticide value, or archive time values. Thus, none were missing the main outcome, exposure predictor, and/or covariate values employed in this paper. Stata ${ }^{\circledR}$ (versions 8.0 and 12.0, College Station, Texas, USA) was used. The 2 -sided $P$ values $<0.05$ designated statistical significance.

\section{Results}

\section{Participant characteristics}

Table 1 shows demographic characteristics for the 80 paired participants, and by case and control status, and in the first and second sets of 20 pairs. Characteristics of the 81st (unpaired) participant are in the table legend. Data for the full set (and broken down by casecontrol status) for the full 81 are provided in Additional file 1: Table S1 (see Additional file 1). The first set of 20 case-control pairs included more Caucasians and was more ethnically homogeneous $(P=0.013)$. Cases were more likely to be married than controls.

Symptoms, as classified by the Kansan criteria, in the cases versus control are shown in Table 2. The military characteristics of the cases are shown in Table 3.

Table 1 Participant characteristics: demographics for matched set of cases and controls

\begin{tabular}{|c|c|c|c|c|c|c|c|c|c|c|c|c|c|}
\hline \multirow[t]{2}{*}{ Characteristics } & \multicolumn{4}{|l|}{ Combined } & \multicolumn{3}{|c|}{ 1st test-set } & \multicolumn{3}{|c|}{ 2nd test-set } & \multicolumn{3}{|l|}{$P$ value } \\
\hline & All $(n=80)$ & $\begin{array}{l}\text { Case } \\
(n=40)\end{array}$ & $\begin{array}{l}\text { Ctrl } \\
(n=40)\end{array}$ & $P$ & $\begin{array}{l}\text { Case } \\
(n=20)\end{array}$ & $\begin{array}{l}\text { Ctrl } \\
(n=20)\end{array}$ & $P$ & $\begin{array}{l}\text { Case } \\
(n=20)\end{array}$ & $\begin{array}{l}\text { Ctrl } \\
(n=20)\end{array}$ & $P$ & All & Case & Ctrl \\
\hline Age (years, mean $\pm S D$ ) & $50 \pm 7.3$ & $50 \pm 7.1$ & $49 \pm 7.5$ & 0.88 & $51 \pm 6.9$ & $51 \pm 7.6$ & 0.81 & $48 \pm 7.2$ & $48 \pm 7.5$ & 0.98 & 0.14 & 0.23 & 0.38 \\
\hline Male (\%) & 93 & 93 & 93 & 1.0 & 90 & 90 & 1.0 & 95 & 95 & 1.0 & 0.40 & 0.55 & 0.55 \\
\hline Married (\%) & 54 & 68 & 40 & 0.014 & 65 & 35 & 0.058 & 70 & 45 & 0.11 & 0.50 & 0.74 & 0.52 \\
\hline \multicolumn{14}{|l|}{ Ethnicity (\%) ${ }^{\mathrm{a}}$} \\
\hline Caucasian & 55 & 55 & 55 & 1.0 & 70 & 70 & 1.0 & 40 & 40 & 1.0 & 0.007 & 0.057 & 0.057 \\
\hline Hispanic & 15 & 15 & 15 & 1.0 & 0 & 0 & 1.0 & 30 & 30 & 1.0 & $<0.001$ & 0.008 & 0.008 \\
\hline African American & 20 & 20 & 20 & 1.0 & 25 & 25 & 1.0 & 15 & 15 & 1.0 & 0.26 & 0.43 & 0.43 \\
\hline Asian & 7.5 & 7.5 & 7.5 & 1.0 & 0 & 0 & 1.0 & 15 & 15 & 1.0 & 0.011 & 0.072 & 0.072 \\
\hline Native American & 2.5 & 2.5 & 2.5 & 1.0 & 5.0 & 5.0 & 1.0 & 0.0 & 0.0 & 1.0 & 0.15 & 0.31 & 0.31 \\
\hline
\end{tabular}

The unmatched $81 \mathrm{st}$ participant (case) was an unmarried, African American male in his $60 \mathrm{~s}$. $P$ values are from $t$-tests of difference in mean (continuous variables) and chi-squared tests (categorical variables)

Ctrl control, SD standard deviation, MDA malondialdehyde

a Designations for specific ethnicities are based on the matched half, where half-matches were used. The first-set (batch) evaluated MDA in the first 20 case-control pairs. The second-set (batch) evaluated the remaining participants 


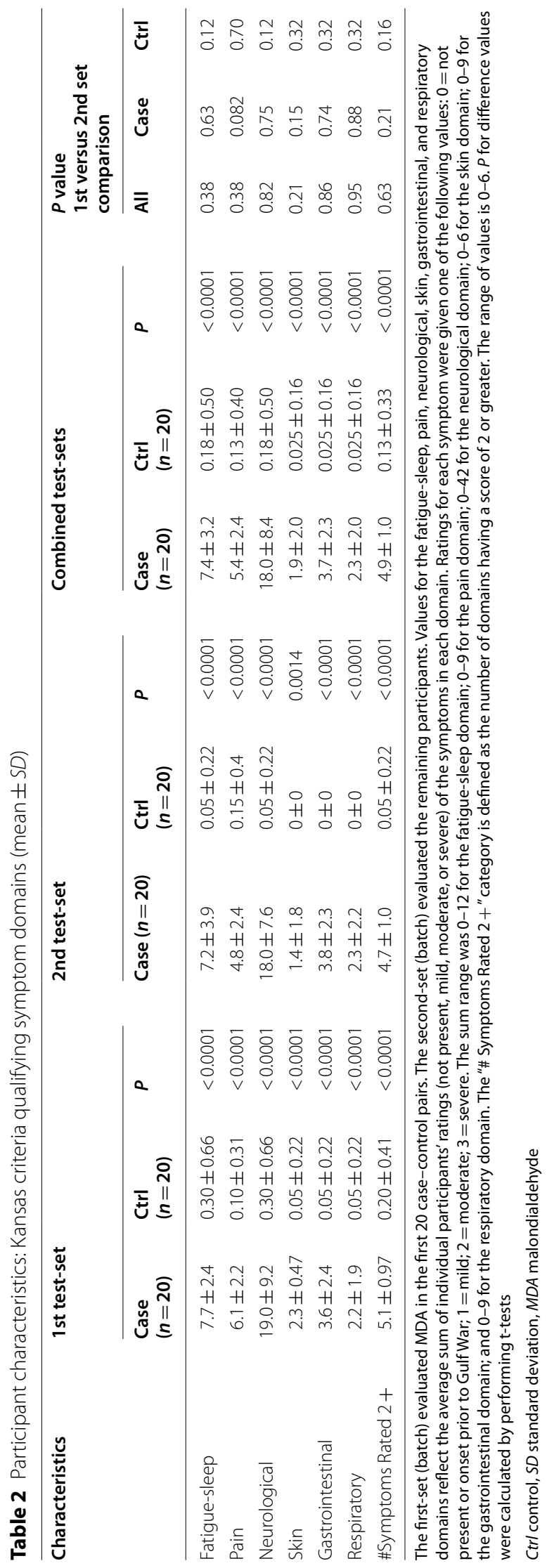


Table 3 Military characteristics: Gulf War veteran subsample

\begin{tabular}{|c|c|c|c|c|}
\hline \multirow[t]{2}{*}{ Characteristics } & \multicolumn{3}{|c|}{ Case vs control comparisons $(n)$} & \multirow{2}{*}{$\begin{array}{l}P \text { value } \\
1 \text { st versus } \\
2 \text { nd test-set } \\
\text { comparison }\end{array}$} \\
\hline & $\begin{array}{l}\text { All } \\
(n=40)\end{array}$ & $\begin{array}{l}1 \text { st test-set } \\
(n=20)\end{array}$ & $\begin{array}{l}2 n d \text { test-set } \\
(n=20)\end{array}$ & \\
\hline Rank & & & & 0.035 \\
\hline Enlisted & 36 & 16 & 20 & \\
\hline Officer & 4 & 4 & 0 & \\
\hline Branch & & & & 0.25 \\
\hline Army & 12 & 5 & 7 & \\
\hline Marine corps & 12 & 4 & 8 & \\
\hline Navy & 11 & 8 & 3 & \\
\hline Air force & 5 & 3 & 2 & \\
\hline \multicolumn{5}{|l|}{ Status } \\
\hline Regular & 35 & 18 & 17 & 0.83 (NS) \\
\hline Reserves & 3 & 1 & 2 & \\
\hline National guard & 2 & 1 & 1 & \\
\hline
\end{tabular}

$P$ values were based on Chi- 2 testing. The first-set (batch) evaluated MDA in the first 20 case-control pairs. The second-set (batch) evaluated the remaining participants

MDA malondialdehyde

\section{MDA comparison}

$\mathrm{MDA}_{1}$ was significantly lower in cases than controls in the full sample, as well as in both first and second sets (Table 4). MDA 1 in the control was lower in the first than the second set. In regression with robust $S E$, lower $\mathrm{MDA}_{1}$ was predicted by case status: case beta $=-5.7(S E=1.80), P=0.002$.

\section{Exposures}

Additional file 1: Table S2 shows each self-rated exposure (\#no-\#yes), in the total sample ("all"), in cases and controls, and in the first and second test-sets (see Additional file 1). Exposure rated as "uncertain" was excluded from the data. The difference between summed yes and no values and 41 (for cases) or 40 (for controls) provides the number that rated the exposure as unsure or (rarely), did not rate the exposure. These are presented as numbers rather than percentages. Exposures include those of potential relevance for the Gulf. Additional file 1: Table S3 shows self-ratings for Gulf-specific exposures (assessed only in Gulf War veterans) (see Additional file 1).

Military-specific exposures were generally asked only of cases (shown in Additional file 1: Table S3). However, we inquired about the history of Botulinum toxoid vaccine in both cases and controls. This vaccine was used in a minority of those who were deployed (though its use has been linked to GWI [22], so participants with GWI, among deployed veterans, may be disproportionately exposed). Consistent with this, only four among the 40 veterans, and none among the 40 controls, reported this exposure-numbers that reassure against widespread overreporting, overall or among affected veterans.

Of note, the first set of cases (and to a lesser extent, controls) had higher fractions exposed to a number of pesticide-related exposures, as well as fuel-solvent and vaccine exposures, than the second set. For example, among controls, there were 8 exposures with the exposed number greater by at least three in the first group, versus one exposure greater by at least 3 in the second.

\section{Relation of MDA to exposures}

Additional file 1: Table S4 shows the prediction of MDA, by candidate individual MDA predictors, after adjustment for archive time (see Additional file 1). Exposures are included if they showed a significant relation to MDA in the full sample. (For the larger set of assessed exposures, see Additional file 1: Table S2.) Two main categories of exposures dominate this table: fuel-solvent-related and pesticide-herbicide-related. Second-generation antipsychotics also appear significant in cases, but this is based on a single case for each of the antipsychotic agents. The herbicide treatment at work variable is also based on a few. There is an additional representation of several vaccines, in cases only.

Table $4 \mathrm{MDA}_{1}$ difference in case versus control

\begin{tabular}{|c|c|c|c|c|}
\hline & $\begin{array}{l}\text { All } \\
(n=80)\end{array}$ & $\begin{array}{l}\text { 1st test-set } \\
(n=40)\end{array}$ & $\begin{array}{l}\text { 2nd test-set } \\
(n=40)\end{array}$ & $\begin{array}{l}P \text { value } \\
1 \text { st versus } 2 \text { nd test-set }\end{array}$ \\
\hline \multicolumn{5}{|l|}{$\mathrm{MDA}_{1}(\mu \mathrm{mol} / \mathrm{L}, \text { mean } \pm S D)^{\mathrm{a}}$} \\
\hline Combined case + Control & $-2.9 \pm 8.5$ & $-4.6 \pm 6.3$ & $-1.1 \pm 10.0$ & 0.064 \\
\hline Cases & $-5.7 \pm 8.4$ & $-6.4 \pm 6.1$ & $-5.1 \pm 10.0$ & 0.63 \\
\hline Controls & $4.9 e-06 \pm 7.7$ & $-2.9 \pm 6.1$ & $2.9 \pm 8.2$ & 0.016 \\
\hline$P$ value & 0.0021 & 0.076 & 0.011 & - \\
\hline
\end{tabular}

Case minus control values. Values are negative because MDA was lower in cases than in controls

${ }^{\mathrm{a}} \mathrm{MDA}_{1}$ is the departure of MDA from values predicted by time-to-test variables and constant-that is, MDA ${ }_{1}$ captures the variation in MDA that cannot be ascribed to time-to-test. In regression with robust $S E, M_{1} A_{1}$ was significantly negatively predicted by case status: $c a s e$ beta $=-5.7(S E=1.80), P=0.002$. The first-set (batch) evaluated MDA in the first 20 case-control pairs. The second-set (batch) evaluated the remaining participants

$S D$ standard deviation, MDA malondialdehyde, MDA, departure of measured MDA from predicted MDA, - No data 
Several additional fuel-solvent and pesticide variables were negative and significant in at least one subcategory (particularly controls) though not in the total sample, and did not meet criteria for inclusion.

Organochlorines and lice treatment were consistently related to lower MDA, with $P \leq 0.001$ in three categories including the total sample, adjusted for case status. Herbicide treatment at the workplace was also related to lower MDA, but the small number that reported the exposure limited the confidence of the finding.

Lindane, an organochlorine, is the primary lice treatment used. Lice treatment was asked separately because it may have been known for its function, rather than by its pesticide class. So, the observed associations may particularly support a powerful negative association of past organochlorine pesticide exposure to MDA.

Additional file 1: Table S5 shows univariable MDA prediction by Gulf-specific exposures, with and without adjustment for the test-set (see Additional file 1). The exposures that predicted MDA with $P<0.1$ on both regressions are shown. Since test-set differences may arise from differences in relevant exposures (more exposures in the first group), the test-set adjusted version is not necessarily more valid. All regression analyses adjust for time-to-test and ln (time-to-test). Only exposures with an apparent relation to MDA are shown. Other than the positive relationship for "saw smoke," negative relationships predominate, related to potential nerve gas attack [or other exposures that may have led the chemical alarms to sound, gas masks or nuclear biological chemical (NBC) protection suits to be donned].

The pesticide exposures are strongly intercorrelated. Fuel and solvent exposures are also strongly correlated to one another and correlated to pesticide exposures.

Table 5 shows the prediction of MDA by the composite pesticide variable. The pesticide variable was significant in all groups-cases, controls, first test-set, second test-set. The fraction exposed to OPs, organochlorines, and lice treatment was $\sim 10,5$, and 2.5 times greater, respectively, in the first relative to the second group (25\%, $12.5 \%$, and $12.5 \%$ in the first set, vs. $9.6 \%, 4.8 \%$, and $2.4 \%$ in the second, respectively). The difference between fractions exposed in the first and second testset was significant: e.g. $P=0.009$ for the presence of at least one such exposure, and $P=0.002$ for OPs specifically. All regressions were performed with adjustment for time-to-test and $\ln$ (time-to-test), unless otherwise stated. Time-to-test variables were significant for controls, and for the total sample adjusted for case status; but did not alter coefficients or significance of the pesticide (composite) variable in any analysis, and did not approach significance for other analyses.

Veterans were also asked about Gulf-specific exposures, and one of these is of potential relevance, given the pesticide finding incorporating OPs. A regression model for cases, assessing variables that cannot be replicated in controls, prioritized exposures tied to potential nerve agent exposure (OP). Inclusion of these variables led to an $R^{2}$ of 0.60 , founded on large negative associations of potential OP (nerve agent) exposure, and positive associations of a composite measure of (history of use of) second generation antipsychotics (2GA) (this includes the three 2nd generation antipsychotics, olanzapine, risperidone, quetiapine [23])-to which, however, few were exposed.

In the two-variable model (with full adjustments for time-to-test, $\ln$ (time-to-test)), test-set-but without the antipsychotic variable), coefficients are identical and $P$ values significant with or without exclusion of those who cite their exposure as "unsure"-but the sample size is reduced to 25 if those citing exposure as "unsure" are excluded. To better support the larger number of adjustment variables, the models showed include those who rated the variable as "unsure." If

Table 5 Prediction of MDA by pesticides (composite variable)

\begin{tabular}{lc}
\hline MDA & $\boldsymbol{\beta}$ (SE) \\
\hline Adjusted for case status and 1st versus 2nd set $(n=81)$ & $-5.3(1.3)$ \\
In case and in control separately adjusted for 1st versus 2nd set & $<0.001$ \\
Case $(n=41)$ & $-5.2(1.7)$ \\
Control $(n=40)$ & $-8.0(1.7)$ \\
In first and in second set separately adjusted for case status & $-4.5(1.3)$ \\
1st set $(n=40)$ & $-7.0(3.0)$ \\
2nd set $(n=41)$ & 0.001
\end{tabular}

Regressions with robust standard errors, adjusted for time-to-test and In(time-to-test). For the composite pesticide variable, exposure was deemed "present" if there was exposure to any of the components; otherwise it was coded as absent. Exposure was present for 11 cases, 6 controls, 13 in the first test-set, and 4 in the second. For the remainder, exposure was absent. The first-set (batch) evaluated MDA in the first 20 case-control pairs. The second-set (batch) evaluated the remaining participants

SE standard error, MDA malondialdehyde 
those rating an exposure as "unsure" in the 3-variable model are excluded, despite the smaller sample size, each predictor remains significant.

Models separately optimized for cases and controls are shown in Additional file 1: Tables S6 and S7, respectively (see Additional file 1). These do not have the authority conferred by replication of findings across cases and controls. The optimized case model includes chemical attacks and composite pesticides as negative predictors.

\section{Discussion}

The current study showed lower plasma MDA concentration in veterans with GWI than in matched healthy controls. Low MDA was predicted by past pesticide exposure (a finding that was separately affirmed in cases and in controls).

MDA is viewed as a biomarker of oxidative stress based on a $\mathrm{CCl}_{4}$ exposure model; and oxidative stress is thought to be a feature of GWI, with findings extending to animal models of GWI (see Additional file 1). Many arachidonic acid products appear to be depressed in veterans with GWI. A preliminary muscle biopsy study [24] supports impaired mitochondrial fatty acid oxidation in GWI, linking MDA with impaired mitochondrial function in this setting.

MDA was depressed in veterans with GWI in both the first and second test-sets in the current study. The association between depressed MDA with past exposures to some classes of pesticides was evident both in veterans with GWI and healthy controls. We underscore that pesticides were used in the Gulf to protect against insect vectors of disease (and indeed, rates of infectious diseases were low during the Gulf relative to many prior conflicts). Qualitative concordance of key findings in cases and controls strengthens confidence in the findings.

In GWI cases, proxies for exposure to OP nerve agent-to which exposure occurred during the Khamisiyah munitions depot demolition and other episodes [25]-predicted lower MDA, that swamped pesticide exposure as a contributory predictor of lower MDA. This, coupled with the larger absolute magnitude of the coefficient for OP exposure (relative to other pesticide exposures) in controls, suggest the possibility that MDA depression may particularly be associated with (past) OP exposure. A model in GWI cases that considered only putative nerve agent related exposure and secondgeneration antipsychotic exposure (which predict in the opposite direction) produced an $\mathrm{R}^{2}$ of 0.60 - that is, it appeared to explain a high fraction of the variance. The antipsychotic findings were based on low numbers-but more than one agent in the class contributed.

In controls, there is less potential for competing and unmeasured exposures to drive spurious exposure-marker relationships. In this group, it is less likely a given exposure serves as a proxy for higher exposure to many unrelated agents, as might be hypothesized for the veteran group. Additionally, there are expected to be fewer other exposures to add variance; and fewer who cited uncertainty about exposures. Unlike the situation for many veterans during the Gulf conflict, controls may often have been in charge of their exposure. This adds relative confidence to findings in controls, in whom each of several pesticide-herbicide agents was independently associated with lower MDA in a multivariable regression model. Greater exposures in the first test-set may explain the apparently lower MDA in control subjects in the first versus second group.

Multiple toxic co-exposure classes in veterans with GWI were correlated with one another, which may add uncertainty to the foundations of exposure relations in Gulf War veterans. Such uncertainty could be attenuated by replication in controls.

A small number of veterans were exposed to secondgeneration antipsychotic drugs-olanzapine, risperidone or quetiapine. These agents are widely used for many non-psychotic conditions. Gulf War veterans may have particular prospects for exposure, both due to more frequent contact with the healthcare system and because treatment guidelines put forth by the Department of Veterans Affairs (VA) for GWI, subsumed under the rubric of chronic multisymptom illness, have endorsed-without supportive evidence-psychiatric medications for this condition [26]. Though our analyses involving these exposures were based on small numbers, providing attendant uncertainty to estimates, the large magnitude of effect (positive prediction of MDA), and consistency across each of the agents (significant whether assessed separately or grouped together), adds modest confidence to the finding. This is consistent with previous reports that these agents are oxidative stressors [27].

Exposures may alter lipids, not just peroxidation of them; and depression or elevation of the lipid can lead to depression or elevation of its oxidized version. This cannot be excluded as a possible factor in the prediction of higher MDA by these agents, and may explain the prediction of lower MDA by pesticides-although actual reductions in OS are an alternate possibility.

Lower MDA levels in veterans with GWI might also reflect altered lipid profiles. Lower MDA and higher 8-hydroxy-2'-deoxyguanosine (8-OHDG) have been shown to predict case status, suggesting these candidate general markers of OS show a dissociation in this setting [28]. MDA derives from arachidonic acid metabolism $[29,30]$. We previously reported widespread depressions in prostaglandins and leukotrienes in veterans with GWI [31]; these are also (eicosanoid) products of arachidonic 
acid [32]. And in a metabolomic assessment, we found depressions in yet other arachidonate-derived products, such as 12-hydroxyeicosatetraenoic acid (12-HETE) and endocannabinoids [33]. Pesticides, as well as fuels and solvents [34], have been reported to alter lipid profiles of membranes. This supports prospects for alterations in lipid profiles in the blood, because membrane phospholipids serve as reservoirs for arachidonic acid and its eicosanoid products [35].

Prior evidence supports lipidomic changes in animal models of GWI that involve exposure to pesticides [36]. Pesticides (and herbicides) are typically delivered in solvents, which may (among other ingredients [37]) influence their effects. Prior evidence supports membrane lipid changes following exposure to solvents, including increases in linoleic acid derivatives in membranes [34], as well as membrane changes and fatty acid metabolism disturbances following pesticide exposure [38].

Lipid changes could serve "adaptive" functions, for instance, in response to acute effects of OS on membrane fluidity or permeability [39], or conversely, as part of protecting against potential arachidonic acid-induced increases in OS and mitochondrial impairment [18]. Reduced arachidonic acid and products could also contribute to health issues, or both (or in principle, neither). For instance, reduced arachidonate in membranes may be tied to membrane fluidity and thereby possibly reduce adenosine triphosphate (ATP) leakage at least in some cell types [39], but this may be tied to less ATP production (albeit conceivably as an adaptive consequence) [16, 17]. Factors tied to greater membrane rigidity are also tied to greater resistance against cytotoxic stressors, so conversely this fluidizing effect may impair resistance [40]. Indeed, it could be speculated that this is a factor in elevated multiple chemical sensitivity (and heightened sensitivity to radiation in a subset of that group-radiation has also been reported to lead to membrane fluidization [15]) in veterans with pesticide exposure [41]. The possibility that effects are adaptive must be considered in the context of the evolutionarily recent and primarily human introduction of the relevant chemicals. Irrespective of these considerations, whether low MDA meaningfully reflects lower OS in this setting, assessed by non-lipid measures, requires further study. There is also the possibility of "oxidative preconditioning" in which prior exposure to an oxidative stressor leads to upregulation of antioxidant defenses which could produce true reductions in oxidative stress $[42,43]$, as the basis for depressed MDA. Finally, veterans with GWI might be taking more antioxidants. However, the lack of depression of other oxidative stress markers (not shown), including total antioxidant capacity (and see prior discussion of 8-OHDG), and presence of depressions of other arachidonic acid derivatives, may render this less likely.

Lipid alterations from OPs, and membrane effects deriving from these, impair mitochondrial ATP production [44]. These might contribute to or underlie the bioenergetic deficits that have been reported in veterans with GWI $[10,11]$. Membrane arachidonic acid relates positively to mitochondrial function $[16,17]$. Thus, the mitochondrial impairment in GWI may portend lower membrane arachidonic acid reservoirs; and moreover, reduced fatty acid beta-oxidation, from bioenergetic impairment in GWI, may reduce the oxidative modification of arachidonic acid [19].

A strength of the current study is the use of the more sensitive Kansas symptom criteria for GWI, as well as the CDC symptom criteria. Also, cases and controls were matched for age, sex and ethnicity. Because veterans were exposed to many agents, providing concern that exposures may serve as proxies for correlated exposures, assessment of exposure relations to MDA in a control group, including some who had exposure to candidate agents, is a strength-and provides important corroboration of key findings.

A key limitation in the current study lies in the fact that exposures were based on recall, and thus subjected to significant recall bias. Also, recruitment was not contingent on the presence or absence of specific exposures. Some controls might be biochemically more similar to cases, if they have had similar exposures. However, the ability to assess exposure relations separately in controls relies on the presence of exposures in some controls. (The second set of participants-including the latter 20 case-control pairs, in whom MDA was assessed separately-included few who cited exposure to assessed pesticides, reducing the ability to assess relations of these exposures to MDA in the second set.) Controls did not have Gulf-specific exposures: analyses here focus on exposures that could be relatively corroborated in controls. Exposures in Gulf War veterans, particularly, may be correlated with other exposures not included in the current study. However, concordance of key findings in controls, where this concern is markedly attenuated, bolsters confidence in key findings. Additionally, among Gulf-specific exposures, ties to MDA were predominantly seen for exposures that are expected to correlate with nerve agent (OP) exposure. The direction of association to MDA was the same for OP pesticide exposures, in cases and controls, providing relative corroboration. Quantitation of the exposure, for many of these exposures of interest, can be difficult.

The study assessed only plasma MDA; tissue MDA (e.g. brain tissue) may not be similarly depressed. Assessment of MDA was conducted well after Gulf 
exposures. This is a potential strength as well as a weakness, since there is particular cause to look at those markers and mechanisms for which alterations are present or sustained well after exposure in veterans with GWI, in whom health issues have been sustained; and to understand long term health effects of exposures. Increases in OS have been reported in the short term, following exposure to both pesticides [45] and solvents $[46,47]$. However, a number of findings underscore that long-term effects (delayed) of OPs and carbamates may differ from short-term effects $[48,49]$.

It is possible that in long-term follow-up after pesticide exposure, lower MDA could arise from actually lower extracellular OS (OS is compartmentalized [50]; mitochondrial OS, for instance, may remain higher). Such an effect could occur from sustained adaptive upregulation of antioxidant defenses, or reduced fatty acid beta-oxidation [19], or conceivably intake of antioxidant supplements. However, the latter would be less able to explain an association of pesticide exposure to lower MDA in healthy controls.

\section{Conclusions}

Past pesticide and OP exposure are associated with long-term depression of MDA, and likely other arachidonic acid products. The finding raises questions about whether MDA and markers of lipid peroxidation are valid markers of OS. Concurrent assessments of markers that do not rely on lipids, such as 8-OHDG, a marker of oxidative injury to deoxyribonucleic acid (DNA); or ratio of oxidized to reduced quantities like glutathione or coenzyme Q10, may be necessary. Attention should also be given to the relation of time after exposure to effect on candidate markers of OS.

\begin{abstract}
Abbreviations
12-HETE: 12-Hydroxyeicosatetraenoic acid; 2GA: Second generation antipsychotic; 8-OHDG: 8-Hydroxy-2'-deoxyguanosine; ATP: Adenosine triphosphate; $\mathrm{CCl}_{4}$ : Carbon tetrachloride; CDC: Centers for disease control and prevention; Ctrl: Control; DNA: Deoxyribonucleic acid; GWI: Gulf War illness; MDA: Malondialdehyde; $\mathrm{MDA}_{1}$ : Departure of measured MDA from predicted MDA; NBC: Nuclear biological chemical; NMPI: N-methyl-2-phenylindole; OP: Organophosphate; OS: Oxidative stress; SD: Standard deviation; SE: Standard error; VA: Department of Veterans affairs.
\end{abstract}

\section{Supplementary Information}

The online version contains supplementary material available at https://doi. org/10.1186/s40779-021-00337-0.

Additional file 1. Additional files The following are available as supplementary data in the Additional files: Table S1 Demographics of study participants $(n=81)$. Table S2 Exposures in cases and controls. Table S3 Gulf-specific exposures in first vs second test-set. Table S4 Exposure predictors of MDA - exposures significant on univariable analysis in the total sample. Table S5 Gulf-specific exposure predictors of MDA - exposures significant on univariable analysis variables assessed for cases only ( $n=$ 41). Table S6 Optimized MDA Model for Cases $(n=41)$. Table S7 MDA multivariable prediction in controls.

\section{Acknowledgements}

We sincerely thank the Gulf War veterans and controls who generously gave their time to this effort, and the study personnel, especially Janis Ritchie BSN, without whom this would not have been possible.

\section{Authors' contributions}

BG designed and oversaw conducted of the study, performed the initial analyses, drafted the initial paper, and participated in the investigation, supervision, project administration and funding acquisition. SD performed the MDA assessments and was involved in revisions of the paper for intellectual content. BY performed analyses in an early stage of the study and participated in review and editing. HK participated in data curation and project administration, was involved in validating statistical results, editing for content and style, and identifying references. AM was involved in validation, writing-review and editing, and administrative functions. JH assisted in restructuring a shorter paper, worked with BG to narrow the scope of the manuscript and rewrite to comport with the reduced scope, conducted validation of the resulting tables, as well as administrative aspects of manuscript submission and revisions. All authors read and approved the final manuscript.

\section{Funding}

The parent study was funded by the Department of Defense Congressionally Directed Medical Research Program (GW093063). The funders had no role in study design, data collection and analysis, decision to publish, or preparation of the manuscript. No additional external funding was received.

\section{Availability of data and materials}

Adequately deidentified data used and/or analyzed during the current study are available from the corresponding author on reasonable request.

\section{Declarations}

Ethics approval and consent to participate

The study and all study material were approved by the University of California, San Diego, Human Research Protections Program (IRB \#100959), as well as the USAMRMC Office of Research Protection. All participants gave written informed consent to participate in the study.

\section{Consent for publication}

Not applicable.

\section{Competing interests}

The authors declare that they have no competing interests.

\section{Author details}

${ }^{1}$ Department of Medicine, School of Medicine, University of California, San Diego, La Jolla, CA 92093, USA. ²Department of Pathology and Immunology, Baylor College of Medicine, Houston, TX 77030, USA. ${ }^{3}$ Swedish Medical Center, Seattle, WA 98109, USA. ${ }^{4}$ Henry M. Jackson Foundation for the Advancement of Military Medicine, San Diego, CA 92134, USA. ${ }^{5}$ Santa Clara Valley Medical Center, San Jose, CA 95128, USA.

Received: 2 February 2021 Accepted: 3 August 2021

Published online: 17 August 2021

\section{References}

1. Kadiiska MB, Gladen BC, Baird DD, Germolec D, Graham LB, Parker CE, et al. Biomarkers of oxidative stress study II: are oxidation products of lipids, proteins, and DNA markers of CCl4 poisoning? Free Radic Biol Med. 2005;38(6):698-710.

2. Binns JH, Barlow C, Bloom FE, Clauw DJ, Golomb BA, Graves JC, et al. Gulf War illness and the health of Gulf War veterans. Scientific findings and 
recommendations. Washington D.C.: U.S. Government Printing Office; 2008.

3. Binns JH, Bloom FE, Bunker JA, Crawford F, Golomb BA, Graves JC, et al. Gulf War illness and the health of GulfWar veterans: research update and recommendations, 2009-2013. Washington, D.C.: U.S. Government Printing Office; 2014.

4. Golomb BA. Acetylcholinesterase inhibitors and Gulf War illnesses. Proc Natl Acad Sci USA. 2008;105(11):4295-300.

5. White RF, Steele L, O'Callaghan JP, Sullivan K, Binns JH, Golomb BA, et al. Recent research on Gulf War illness and other health problems in veterans of the 1991 Gulf War: effects of toxicant exposures during deployment. Cortex. 2016;74:449-75. https://doi.org/10.1016/j.cortex.2015.08. 022.

6. Fricker RD, Reardon E, Spektor DM, Cotton SK, Hawes-Dawson J, Pace JE, et al. Pesticide use during the Gulf War: a survey of Gulf War veterans. Santa Monica, CA: RAND; 2000.

7. Cecchine G, Golomb BA, Hilborne LH, Spektor DM, Anthony RA. A review of the scientific literature as it pertains to Gulf War illnesses, Vol 8: Pesticides. Santa Monica: RAND; 2000.

8. Sullivan K, Krengel M, Bradford W, Stone C, Thompson TA, Heeren T, et al. Neuropsychological functioning in military pesticide applicators from the Gulf War: effects on information processing speed, attention and visual memory. Neurotoxicol Teratol. 2018;65:1-13. https://doi.org/10.1016/j.ntt. 2017.11.002.

9. Golomb BA. Oxidative stress and mitochondrial injury in chronic multisymptom conditions: from Gulf War illness to autism spectrum disorder. Nat Prec. 2012. https://doi.org/10.1038/npre.2012.6847.1.

10. Koslik HJ, Hamilton G, Golomb BA. Mitochondrial dysfunction in Gulf War illness revealed by 31 phosphorus magnetic resonance spectroscopy: a case-control study. PLoS ONE. 2014;9(3):e92887.

11. Chen Y, Meyer JN, Hill HZ, Lange G, Condon MR, Klein JC, et al. Role of mitochondrial DNA damage and dysfunction in veterans with Gulf War illness. PLoS ONE. 2017;12(9):e0184832.

12. Wei YH. Mitochondrial DNA mutations and oxidative damage in aging and diseases: an emerging paradigm of gerontology and medicine. Proc Natl Sci Counc Repub China B. 1998;22(2):55-67.

13. Yakymenko I, Tsybulin O, Sidorik E, Henshel D, Kyrylenko O, Kyrylenko S. Oxidative mechanisms of biological activity of low-intensity radiofrequency radiation. Electromagn Biol Med. 2015;35(2):186-202.

14. Bhatia AL, Manda K. Study on pre-treatment of melatonin against radiation-induced oxidative stress in mice. Environ Toxicol Pharmacol. 2004;18(1):13-20.

15. Wang C, Cong J, Xian H, Cao X, Sun C, Wu K. The effects of electromagnetic pulse on fluidity and lipid peroxidation of mitochondrial membrane. Zhonghua Lao Dong Wei Sheng Zhi Ye Bing Za Zhi. 2002;20(4):266-8. https://doi.org/10.3760/cma.j.issn.1001-9391.2002.04. 009.

16. Khairallah RJ, Kim J, O'Shea KM, O'Connell KA, Brown BH, Galvao T, et al. Improved mitochondrial function with diet-induced increase in either docosahexaenoic acid or arachidonic acid in membrane phospholipids. PLOS ONE. 2012;7(3):e34402.

17. Arai M, Gordon ER, Lieber CS. Decreased cytochrome oxidase activity in hepatic mitochondria after chronic ethanol consumption and the possible role of decreased cytochrome aa3 content and changes in phospholipids. Biochim Biophys Acta. 1984;797(3):320-7.

18. Cocco T, Di Paola M, Papa S, Lorusso M. Arachidonic acid interaction with the mitochondrial electron transport chain promotes reactive oxygen species generation. Free Radic Biol Med. 1999;27(1-2):51-9. https://doi. org/10.1016/s0891-5849(99)00034-9.

19. Chen CT, Trepanier MO, Hopperton KE, Domenichiello AF, Masoodi M, Bazinet RP. Inhibiting mitochondrial beta-oxidation selectively reduces levels of nonenzymatic oxidative polyunsaturated fatty acid metabolites in the brain. J Cereb Blood Flow Metab. 2014;34(3):376-9.

20. Fukuda K, Nisenbaum R, Stewart G, Thompson WW, Robin L, Washko RM, et al. Chronic multisymptom illness affecting Air Force veterans of the Gulf War. JAMA. 1998:280(11):981-8.

21. Steele L. Prevalence and patterns of Gulf War illness in Kansas veterans: association of symptoms with characteristics of person, place, and time of military service. Am J Epidemiol. 2000;152(10):992-1002.

22. Gray GC, Reed RJ, Kaiser KS, Smith TC, Gastanaga VM. Self-reported symptoms and medical conditions among 11,868 Gulf War-era veterans: the
Seabee Health Study. Am J Epidemiol. 2002;155(11):1033-44. https://doi. org/10.1093/aje/155.11.1033.

23. Heres S, Davis J, Maino K, Jetzinger E, Kissling W, Leucht S. Why olanzapine beats risperidone, risperidone beats quetiapine, and quetiapine beats olanzapine: an exploratory analysis of head-to-head comparison studies of second-generation antipsychotics. Am J Psychiatry. 2006;163(2):185-94.

24. Fung A, Koslik H, Ritchie J, Dinkeloo E, Golomb BA. Bioenergetics in veterans with Gulf War illness versus healthy controls; replication and expansion. In: Gulf War illness state of the science virtual conference: August 19 2020; Washington, D.C.; 2020.

25. General Accounting Office. Gulf War illnesses: DoD's conclusions about U.S. troops' exposure cannot be adequately supported. GAO report number GAO-04-159. 2004. https://www.gao.gov/assets/gao-04-159.pdf. Accessed 9 July 2021.

26. Department of Veterans Affairs. VA/DoD clinical practice guideline for the management of chronic multisymptom illness. 2014. https://www.healt hquality.va.gov/guidelines/MR/cmi/NADoDCMICPG.pdf. Accessed 9 July 2021.

27. Walss-Bass C, Weintraub ST, Hatch J, Mintz J, Chaudhuri AR. Clozapine causes oxidation of proteins involved in energy metabolism: a possible mechanism for antipsychotic-induced metabolic alterations. Int J Neuropsychopharmacol. 2008;11(8):1097-104.

28. Golomb BA. Metabolomic and lipidomic biomarkers of Gulf War illness. Research advisory committee on Gulf War veterans' illnesses. 2015, Sept 28, Washington, D.C. https://www.va.gov/RAC-GWVI/meetings/sep2015/ RAC-AgendaSept2015.pdf. Accessed 9 Jul 2021.

29. Maes M, Galecki P, Chang YS, Berk M. A review on the oxidative and nitrosative stress (O\&NS) pathways in major depression and their possible contribution to the (neuro)degenerative processes in that illness. Prog Neuropsychopharmacol Biol Psychiatry. 2011:35(3):676-92.

30. de Gaetano G, Rajtar G, Livio M, Merino J. Arachidonic acid-induced malondialdehyde formation in rat platelets. Kinetic aspects and inhibition by acetylsalicylic acid and indomethacin. Naunyn Schmiedebergs Arch Pharmacol. 1980;312(1):85-9.

31. Golomb BA, Koslik HJ, Christians U, Ritchie J, Wilson P, Elkins N, et al. Depressed prostaglandins and leukotrienes in veterans with Gulf War illness. J Environ Sci Health B. 2019;54(8):623-39. https://doi.org/10.1080/ 03601234.2019.1596001.

32. Poole MD, Pillsbury HC 3rd. Prostaglandins and other metabolites of arachidonic acid. An overview for the otolaryngologist. Arch Otolaryngol. 1985;111(5):317-21. https://doi.org/10.1001/archotol.1985.0080007006 9010.

33. Naviaux RK, Naviaux JC, Li K, Wang L, Monk JM, Bright AT, et al. Metabolic features of Gulf War illness. PLoS ONE. 2019;14(7):e0219531.

34. Kyrklund T, Kjellstrand P, Haglid KG. Membrane lipid changes in organic solvent tolerant neural cells. Arch Toxicol Suppl. 1986;9:38-45.

35. Farooqui AA, Horrocks LA, Farooqui T. Glycerophospholipids in brain: their metabolism, incorporation into membranes, functions, and involvement in neurological disorders. Chem Phys Lipids. 2000;106(1):1-29.

36. Abdullah L, Evans JE, Joshi U, Crynen G, Reed J, Mouzon B, et al. Translational potential of long-term decreases in mitochondrial lipids in a mouse model of Gulf War illness. Toxicology. 2016;372:22-33. https://doi.org/10. 1016/j.tox.2016.10.012.

37. Mesnage R, Defarge N, Spiroux de-Vendômois J, Séralini GE. Major pesticides are more toxic to human cells than their declared active principles. Biomed Res Int. 2014;2014:179691. https://doi.org/10.1155/2014/179691.

38. Liu Q, Wang Q, Xu C, Shao W, Zhang C, Liu H, et al. Organochloride pesticides impaired mitochondrial function in hepatocytes and aggravated disorders of fatty acid metabolism. Sci Rep. 2017;7:46339. https://doi.org/ 10.1038/srep46339.

39. Nagasaka R, Okamoto N, Ushio H. Partial oxidative-stress perturbs membrane permeability and fluidity of fish nucleated red blood cells. Comp Biochem Physiol C Toxicol Pharmacol. 2004;139(4):259-66.

40. Lucken-Ardjomande HS. Cholesterol, cardiolipin, and mitochondria permeabilisation. Anticancer Agents Med Chem. 2012;12(4):329-39.

41. Reid S, Hotopf M, Hull L, Ismail K, Unwin C, Wessely S. Multiple chemical sensitivity and chronic fatigue syndrome in British Gulf War veterans. Am J Epidemiol. 2001;153(6):604-9. 
42. León OS, Menéndez S, Merino N, Castillo R, Sam S, Pérez L, et al. Ozone oxidative preconditioning: a protection against cellular damage by free radicals. Mediators Inflamm. 1998;7(4):289-94.

43. Candelario-Jalil E, Mohammed-Al-Dalain S, Fernández OS, Menéndez S, Pérez-Davison G, Merino N, et al. Oxidative preconditioning affords protection against carbon tetrachloride-induced glycogen depletion and oxidative stress in rats. J Appl Toxicol. 2001;21(4):297-301.

44. Videira RA, Antunes-Madeira MC, Madeira VM. Ethylazinphos interaction with membrane lipid organization induces increase of proton permeability and impairment of mitochondrial bioenergetic functions. Toxicol Appl Pharmacol. 2001;175(3):209-16.

45. Abdollahi M, Ranjbar A, Shadnia S, Nikfar S, Rezaie A. Pesticides and oxidative stress: a review. Med Sci Monit. 2004;10(6):RA141-7.

46. Jyothi K, Kalyani D, Nachiappan V. Effect of acute exposure of N, $\mathrm{N}$-dimethylformamide, an industrial solvent on lipid peroxidation and antioxidants in liver and kidney of rats. Indian J Biochem Biophys. 2012;49(4):279-84.
47. Campos FC, Victorino VJ, Martins-Pinge MC, Cecchini AL, Panis C, Cecchini $R$. Systemic toxicity induced by paclitaxel in vivo is associated with the solvent cremophor EL through oxidative stress-driven mechanisms. Food Chem Toxicol. 2014;68:78-86. https://doi.org/10.1016/j.fct.2014.03.013.

48. Somani SM, Husain K, Asha T, Helfert R. Interactive and delayed effects of pyridostigmine and physical stress on biochemical and histological changes in peripheral tissues of mice. J Appl Toxicol. 2000;20(4):327-34.

49. Henderson RF, Barr EB, Blackwell WB, Clark CR, Conn CA, Kalra R, et al. Response of rats to low levels of sarin. Toxicol Appl Pharmacol. 2002;184(2):67-76.

50. Jones DP, Go YM. Redox compartmentalization and cellular stress. Diabetes Obes Metab. 2010;12(Suppl 2):116-25. https://doi.org/10.1111/j. 1463-1326.2010.01266.x.
Ready to submit your research? Choose BMC and benefit from:

- fast, convenient online submission

- thorough peer review by experienced researchers in your field

- rapid publication on acceptance

- support for research data, including large and complex data types

- gold Open Access which fosters wider collaboration and increased citations

- maximum visibility for your research: over 100M website views per year

At BMC, research is always in progress.

Learn more biomedcentral.com/submissions 